\title{
Differences in Energy Expenditure and Typing Speed While Sitting, Standing, and Walking in College Students
}

Original Research

\begin{abstract}
Fred Miller III ${ }^{1}$, Avniel Barrow ${ }^{1}$, Adrian Perez ${ }^{1}$, Yenly Londono Calle ${ }^{1}$
${ }^{1}$ Huntington University, Huntington, Indiana/USA

Open Access

Abstract

Introduction: The purpose of this study was to determine if caloric expenditure and typing speed differed among three positions (sitting, standing, walking).

Published: October 8, 2021

Methods: Participants included 40 college students (18-22 years, 30 males and 10 females) on either the baseball or track and field teams. Each participant was tested for 5 minutes in three different positions. Caloric expenditure was measured via indirect calorimetry and typing productivity via a 3-minute typing test. Repeated measures ANOVAs and T-Tests were performed to determine statistical differences for caloric expenditure and typing speed.

Results: Caloric expenditure (calories per 5 minutes) was significantly higher for walking $(16.4 \pm 3.1)$ than for sitting $(9.0 \pm 2.4, \mathrm{p}<0.0001)$ and standing $(9.4 \pm 2.0, \mathrm{p}$ $<0.0001)$. For typing productivity, standing resulted in faster typing speed than walking (37.4 \pm 10.2 vs. $34.7 \pm 10.7 \mathrm{wpm}, \mathrm{p}=0096)$.

Conclusions: Using a standup walking desk to type while working expends significantly more calories than typing while sitting or standing at a desk. However, typing speed was significantly higher while standing at a desk than while walking at a desk.
\end{abstract}

Key Words: Calories, workstation, productivity

Corresponding author: Fred Miller III, fmiller@huntington.edu

Nutrition: 2021, Volume

4 (Issue 4): 18

ISSN: $2640-2572$

\section{Introduction}

Adults spend an average seven to nine hours per day sitting ${ }^{1}$. Among college students, the time spent in sedentary behaviors has been reported even higher, at 11.88 hours per day ${ }^{2}$. Similarly, a systematic review that included data from twenty three studies also reported a mean of sedentary time among undergraduate students of 11.10 hours measured by domain specific questionaries and 10.69 hours per day measured by accelerometers ${ }^{3}$. Current research concluded that there is a need for research and interventions focused on reducing sedentary time among undergraduate students ${ }^{3}$. Concerns though have been raised about possible negative effects on cognitive performance while walking. One previous study concluded that cognitive performance was not impaired by walking at a self-selected pace and that offices and classrooms should consider implementing active workstations ${ }^{4}$. Another study found typing performance while walking at a moderate speed $(2.25 \mathrm{~km} / \mathrm{hr})$ was similar to typing performance while sitting, however, typing performance was worse while walking at slower $(1.3 \mathrm{~km} / \mathrm{hr})$ and 
faster $(3.2 \mathrm{~km} / \mathrm{hr})$ speeds ${ }^{5}$. Since many college students use laptops for typing notes and searching the internet, additional research on how active workstations may impact cognitive and typing performance would be beneficial.

It is a well-known fact that we sit for prolonged periods of time, and recent research reveals detrimental health effects 3,6,7. Sitting too much has been associated with increased risk of mortality, cardiovascular disease, cancer, hypertension, overweight/obesity, and Type 2 diabetes ${ }^{8-10}$. In addition, other studies have reported the negative health effects of sitting, regardless of meeting the physical activity ACSM guidelines ${ }^{8,11}$. In fact, one of the major recommendations from the updated "Physical Activity Guidelines for Americans" is to move more, sit less ${ }^{12}$.

A variety of methods have been suggested to reduce time spent sitting during our waking hours. For example, some health experts recommend just getting up and moving every 30 to 45 minutes ${ }^{13,14}$. However, this advice still results in a lot of time sitting. In addition, a recent study among faculty and college students about the problem of prolonged sitting on the classroom proposed interventions to reduce bouts of prolonged sitting. These interventions include faculty prompts, modification of pedagogy, educational campaigns and structural changes to classroom designs ${ }^{15}$. In this study, the focus was on improving classroom design by including active workstations. Active workstations are desks that enable one to stand, walk, or cycle while working at a desk/computer. They include standing desks, treadmill desks, and cycling desks. The goal is simple: reduce time spent sitting to improve one's health and possibly improve productivity (or, at least, not affect productivity negatively). Research presents numerous benefits to using active workstations versus sitting. Benefits include more calories burned, reduced boredom (more engagement), better mood and more energy, elevated productivity, improved memory, and increased lifespan ${ }^{16-19}$. In a recent college classroom study, researchers reported that just three weeks of standing (vs. sitting) significantly improved cardiometabolic risk factors ${ }^{16}$. In addition, a recent study looking at the effects of treadmill workstations found improvements in work performance, interactions with coworkers, and daily total physical activity ${ }^{20}$. Some concerns have been raised about negative effects on work performance if one tries to walk on a treadmill during work. Some studies report that performance on cognitive tasks are not impaired during treadmill walking ${ }^{4,21}$. In fact, one study reported treadmill workstations actually improve work performance ${ }^{20}$. Another recent study reported delayed positive effects on attention and recall from walking on a treadmill versus sitting ${ }^{22}$. The primary goal of treadmill workstations is to reduce sedentary time and improve overall health. However, other benefits, such as improved mood, cognition, and productivity, may result.

The purpose of this study was to determine the caloric expenditure and typing speed among three positions (sitting, standing, and walking) in a group of male and female college students. The colleges students were part of the baseball and track teams. This was a convenience sample because one of the authors of this study is part of the track team and the other one was on the baseball team. There is a limited number of athletes on those teams, and only 42 of them agreed to participate on the study. It was hypothesized that there would be a significant difference in caloric expenditure among the three positions and secondly, that typing productivity would not be significantly impacted.

\section{Scientific Methods}

Participants

Forty-two college students were recruited from the baseball and track teams to participate in this study. Of those 42, two could not participate, one due to scheduling conflicts and the other because of medical reasons. Thus, there were 40 participants who participated in testing. After arriving for testing, each participant received and read an informed consent which explained the purpose, method, benefits, and risks of the study. Then after given the opportunity to ask questions, participants agreed to participate by signing the consent form. The study was approved by the Huntington University Institutional Review Board (IRB) prior to any testing.

Protocol

The study was conducted between October 2019 and November 2019. Testing took place on the university campus in the Human Performance Lab. Prior to reporting to the lab, participants were instructed to refrain from vigorous physical activity at least 24 hours before testing, and told not to consume caffeine the day of, or the day before being tested, and directed to refrain from eating or drinking anything (except for water) at least three hours before testing. Each participant was asked prior to testing whether they followed these guidelines. If they did not follow these guidelines, they were not tested and instructed to schedule another time for testing. Many athletes were tested during the weekend when they did not engage in training or competition. 
After following the pre-testing instructions, the participant's bodyweight was measured by a digital scale. Height was measured by a stadiometer. Height and weight measures were then entered in the Parvo Medics True One Metabolic System. Because these measures are important in calculating energy expenditure, it was deemed important to measure weight and height rather than have participants' self-report. Next, the participant received explanation that the VO2 testing would happen in the standing, sitting, and walking $(1.5 \mathrm{mph})$ positions. The slow walking speed was chosen for safety reasons. Participants did not have a practice period, so walking at a slow pace would help prevent falls. In addition, another study was conducted using this speed 23. After explaining the VO2 testing, the headgear and mouthpiece were positioned on the participant. The participant then spent five minutes in each position while VO2 (i.e., energy expenditure) was measured (via Parvo medics True One 2400 Metabolic System). At the beginning of each position and during the first three minutes, participants performed a 3-minute online typing test (www.typingtest.com) on a laptop computer. After completing the 3-minute typing test, three results appeared (typing speed, errors, and adjust speed). The adjusted speed, which accounts for errors, was used to determine typing productivity for each participant in each position.

In this study, half of the participants first position was sitting, then standing, and finally walking, while the other half of participants first position was walking, followed by standing, and then sitting. Participants were instructed to breath normally and not talk.

Statistical Analysis

Descriptive statistics (i.e., means and standard deviations) on the data collected were performed in Excel. Two separate repeated measures ANOVAs performed in Excel were used to determine statistical main effects among the three positions for energy expenditure and typing speed. The Bonferroni correction post hoc analysis was used to determine statistical differences among the three positions for energy expenditure and typing speed. Statistical significance was set at $\mathrm{p} \leq 0.05$ for ANOVA testing and $\mathrm{p} \leq 0.016$ for the Bonferroni correction.

\section{Results}

The participant characteristics are presented in Table 1.

Table 1. Characteristics of the participants.

\begin{tabular}{llll}
\hline & All $(\mathrm{n}=40)$ & Male $(\mathrm{n}=30)$ & Female $(\mathrm{n}=10)$ \\
\hline Age $(\mathrm{yrs})$ & $20.0 \pm 0.4$ & $20.0 \pm 1.3$ & $20.1 \pm 1.0$ \\
Height $(\mathrm{cm})$ & $176.8 \pm 9.7$ & $180.3 \pm 8.1$ & $166.2 \pm 5.5$ \\
Weight $(\mathrm{kg})$ & $78.9 \pm 12.7$ & $82.8 \pm 10.6$ & $67.1 \pm 11.3$ \\
\hline
\end{tabular}

Note: values are Mean $\pm \mathrm{SD}$

Descriptive data for energy expenditure and typing speed are displayed in Table 2. The ANOVA revealed a significant main effect $(p<0.0001)$ in the energy expenditures among the three positions. Post-hoc analysis showed significant differences between sitting and walking and between standing and walking (Table 2). For typing speed, the ANOVA revealed a significant main effect among the three positions $(p=0.0096)$. Post-hoc analysis showed a significant difference between standing and walking (Table 2). Typing speed was not significantly different (based on $\mathrm{p} \leq 0.016$ for the Bonferroni correction) between sitting and standing $(\mathrm{p}=0.927)$ or between sitting and walking $(\mathrm{p}=0.038)$.

Table 2. Energy expenditure and typing speeds in each position.

\begin{tabular}{llll}
\hline & sitting & standing & walking \\
\hline Energy Expenditure (cal/5min) & $9.0 \pm 2.4$ & $9.4 \pm 2.0$ & $16.4 \pm 3.1^{*}$ \\
Typing Speed (words/min) & $37.3 \pm 10.3$ & $37.4 \pm 10.2^{* *}$ & $34.7 \pm 10.7^{* *}$ \\
\hline
\end{tabular}

Note: values are Means \pm SD. $\mathrm{N}=40$.

$*$ Energy expenditure significantly greater in walking than sitting and standing, $p<0.0001$

${ }^{* *}$ Typing speed significantly greater in standing than walking, $p=0.0096$

\section{Discussion}

The primary hypothesis was partially correct as walking did expend more calories than sitting and standing. However, standing did not significantly expend more calories than sitting. On average, walking for five minutes expended about 16.0 calories $(3.2$ calories $/ \mathrm{min}$ ), while sitting and standing expended 9.0 (1.8 calories $/ \mathrm{min})$ and 9.4 (1.9 calories $/ \mathrm{min})$, respectively. Walking expended approximately $78 \%$ and $70 \%$ more calories than sitting and standing, respectively. 
The secondary hypothesis was partially correct in that no significant differences was found between sitting and standing or sitting and walking. However, standing compared to walking did result in approximately $8 \%$ more words typed per minute. On average, participants typed $37.3,37.4$, and 34.7 words per minute while sitting, standing, and walking, respectively.

A previous study ${ }^{24}$ focused on office tasks such as sitting and standing, also reported that energy expenditure during walking was significantly greater than sitting and standing, and that sitting and standing were not significantly different. Furthermore, a recent study reported no differences in energy expenditure between sitting and standing ${ }^{25}$. Both studies suggest, similar to this study, that to increase energy expenditure, standing (versus sitting) is not enough and that one needs to move (walk). Another study comparing the effects of different activities while sitting, standing, and walking, found some sitting activities to yield equal or higher energy expenditure than standing still, and furthermore that increasing walking speeds (from 0.2 to $1.6 \mathrm{mph}$ ) resulted in higher values than both sitting and standing ${ }^{26}$. Previous studies, along with this study, showed that standing is insufficient, and walking (even at a slow pace) is necessary for additional caloric expenditure over sitting. However, in a recent meta-analysis, researchers suggested that even a minimal difference in energy expenditure $(0.15 \mathrm{kcal} / \mathrm{min})$ between sitting and standing could be sufficient in preventing weight gain ${ }^{27}$.

Funk et al. ${ }^{5}$ found typing performance was similar when sitting and walking at $2.25 \mathrm{~km} / \mathrm{hr}$. However, the researchers also found typing while walking at slower $(1.3 \mathrm{~km} / \mathrm{hr})$ and faster $(3.2 \mathrm{~km} / \mathrm{hr})$ speeds were detrimental to typing performance. Although the walking speed in the current study was slightly faster $(2.4 \mathrm{~km} / \mathrm{hr}$ or $1.5 \mathrm{mph}$ compared to $2.25 \mathrm{~km} / \mathrm{hr}$ ), no significant differences were found in typing speeds between typing while seated versus typing while walking. Furthermore, Ehmann et al. ${ }^{21}$ found that self-selected low-intensity walking did not affect executive function, while Alderman et al. ${ }^{4}$ found self-selected walking speed did not impact cognitive tasks, including executive processes. Given these studies' findings, along with the current study's results, it appears walking at either a self-selected or moderate speed (e.g., $2.4 \mathrm{~km} / \mathrm{hr}$ ) would not negatively impact typing or cognitive performance.

This study had three known limitations. First, the population consisted of young college athletes, so the findings cannot be generalized to other populations. Also, findings from this study also may differ in other populations. For example, non-athletes, athletes in other sports, or older participants may show different results. Second, the participants were not given a "practice period" for typing while walking on the treadmill. Most participants had never tried to walk on a treadmill and type on a laptop at the same time. There is typically a learning curve involved that will result in participants getting better with more practice. However, this study did not provide a practice period prior to testing subjects energy expenditure and typing productivity. Therefore, the lower mean score for adjusted typing speed during the walking position may be higher if given a "practice period" prior to testing, which could likely result in a similar mean to the other two positions (sitting and standing). Third, the standing position likely benefitted from a learning curve since it was the second position across the two groups. Randomizing across six groups instead of two would have been a better study design.

This study has three major strengths. First, this study included a three-minute typing test to determine if typing productivity would differ among each position. Many studies only measured energy expenditure and not the effects of work productivity. This study measured both. Second, this study included testing of energy expenditure and typing productivity in three positions (i.e., sitting, standing, walking). The measure of three positions allows for additional direct comparisons than only including two positions found in several studies (e.g., sitting vs. standing or standing vs. walking). Third, this study measured energy expenditure using indirect calorimetry. Because the energy expenditure measured in this study is based on a "gold-standard", these findings are likely more accurate than findings based on other non "gold-standard" techniques utilized in previous studies.

There are two implications from this study. First, this study was able to capture participants that were around the same age and body weight to determine energy expenditure. Second, one of the reasons that the typing portion was included in this study was because many desk workers and even college students usually sit most of the day typing. We were able to demonstrate that office workers whose job may include typing are able to continue their typing productivity while walking on a treadmill, while reducing sedentary behavior. It is well known that sedentary behavior like sitting for several hours can later lead to obesity or other health related diseases ${ }^{7}$. In addition the findings of this study may be generalized to young men that do office type work jobs such as students sitting on a class or lab, working at home typing a paper or composing emails. 
In this study, walking expended the most calories per five minutes (16.4). Sitting and standing resulted in similar energy expenditures (9.0 and 9.4). In addition, walking did not significantly decrease the typing productivity measured by a three-minute typing test when compared with sitting. Based on this study's findings, adding a treadmill workstation in place of a traditional sitting desk or standing desk will lead to greater energy expenditure while possibly maintaining typing productivity with sufficient practice. Furthermore, the greater energy expenditure using a treadmill workstation may help prevent many health issues (e.g., obesity, diabetes) in those workers (e.g., office workers) who currently sit for several hours per day. It is suggested that future studies be performed among different populations such as middle age, women and older populations who perform office work. These populations who currently have a "sitting" job would benefit most from these studies. In addition, incorporation of other work productivity measures (e.g., reading comprehension) and other possible benefits (e.g., improvements in diabetes management and blood pressure) on the affects among the three positions (i.e., sitting, standing, and walking) is warranted. Finally, future studies on the effects that different walking speeds may have on energy expenditure and typing speed is suggested.

\section{Conclusions}

Typing while at a walking desk expends significantly more calories than typing while sitting or standing at a desk, however, typing while standing resulted in a significantly higher typing speed than typing while walking.

\section{Acknowledgements}

The authors would like to thank the Huntington University Kinesiology Department for the use of lab space and equipment. We would also like to thank the participants.

\section{References}

1. Clark B, Sugiyama T. Prevalence, trends, and correlates of sedentary behavior. In: Kanosue K, editor. Physical Activity, Exercise, Sedentary Behavior and Health. Springer. Published online 2018.

2. Moulin MS, Irwin JD. An Assessment of Sedentary Time Among Undergraduate Students at a Canadian University. Published online 2017:14.

3. Moulin MS, Truelove S, Burke SM, Irwin JD. Sedentary time among undergraduate students: A systematic review. Journal of American College Health. 2019;0(0):1-8. doi:10.1080/07448481.2019.1661422

4. Alderman BL, Olson RL, Mattina DM. Cognitive function during low-intensity walking: a test of the treadmill workstation. J Phys Act Health. 2014;11(4):752-758. doi:10.1123/jpah.2012-0097

5. Funk RE, Taylor ML, Creekmur CC, Ohlinger CM, Cox RH, Berg WP. Effect of walking speed on typing performance using an active workstation. Percept Mot Skills. 2012;115(1):309-318. doi:10.2466/06.23.26.PMS.115.4.309-318

6. Chau JY, Reyes-Marcelino G, Burnett AC, Bauman AE, Freeman B. Hyping health effects: a news analysis of the "new smoking" and the role of sitting. Br J Sports Med. 2019;53(16):1039-1040. doi:10.1136/bjsports-2018099432

7. Vallance JK, Gardiner PA, Lynch BM, et al. Evaluating the Evidence on Sitting, Smoking, and Health: Is Sitting Really the New Smoking? Am J Public Health. 2018;108(11):1478-1482. doi:10.2105/AJPH.2018.304649

8. Biswas A, Oh PI, Faulkner GE, et al. Sedentary time and its association with risk for disease incidence, mortality, and hospitalization in adults: a systematic review and meta-analysis. Ann Intern Med. 2015;162(2):123-132. doi:10.7326/M14-1651

9. Guo C, Zhou Q, Zhang D, et al. Total sedentary behavior and TV viewing with risk of overweight/obesity, type 2 diabetes, and hypertension: a dose-response meta-analysis. Diabetes Obes Metab. Published online August 29, 2019. doi: $10.1111 /$ dom. 13867

10. Nicolo ML, Shewokis PA, Boullata J, et al. Sedentary behavior time as a predictor of hemoglobin A1c among adults, 40 to 59 years of age, living in the United States: National Health and Nutrition Examination Survey 2003 to 2004 and 2013 to 2014. Nutr Health. Published online September 25, 2019:260106019870436. doi:10.1177/0260106019870436

11. Even if you exercise, too much sitting is bad for your heart. Mayo Clin Health Lett. 2011;29(9):4.

12. Physical Activity Guidelines - health.gov.

13. Dunstan: Too much sitting-a health hazard - Google Scholar.

14. Cooley D, Pedersen S. A Pilot Study of Increasing Nonpurposeful Movement Breaks at Work as a Means of Reducing Prolonged Sitting. Journal of Environmental and Public Health. 2013;2013:e128376. doi:10.1155/2013/128376 
15. Cowgill BO, Perez V, Gerdes E, et al. Get up, stand up, stand up for your health! Faculty and student perspectives on addressing prolonged sitting in university settings. Journal of American College Health. 2021;69(2):198-207. doi:10.1080/07448481.2019.1661419

16. Butler KM, Ramos JS, Buchanan CA, Dalleck LC. Can reducing sitting time in the university setting improve the cardiometabolic health of college students? Diabetes Metab Syndr Obes. 2018;11:603-610. doi:10.2147/DMSO.S179590

17. Gilson ND, Hall C, Renton A, Ng N, von Hippel W. Do Sitting, Standing, or Treadmill Desks Impact Psychobiological Indicators of Work Productivity? J Phys Act Health. 2017;14(10):793-796. doi:10.1123/jpah.2016-0712

18. Jerome M, Janz KF, Baquero B, Carr LJ. Introducing sit-stand desks increases classroom standing time among university students. Prev Med Rep. 2017;8:232-237. doi:10.1016/j.pmedr.2017.10.019

19. Winkler EAH, Chastin S, Eakin EG, et al. Cardiometabolic Impact of Changing Sitting, Standing, and Stepping in the Workplace. Med Sci Sports Exerc. 2018;50(3):516-524. doi:10.1249/MSS.0000000000001453

20. Ben-Ner A, Hamann DJ, Koepp G, Manohar CU, Levine J. Treadmill Workstations: The Effects of Walking while Working on Physical Activity and Work Performance. PLoS One. 2014;9(2). doi:10.1371/journal.pone.0088620

21. Ehmann PJ, Brush CJ, Olson RL, Bhatt SN, Banu AH, Alderman BL. Active Workstations Do Not Impair Executive Function in Young and Middle-Age Adults. Med Sci Sports Exerc. 2017;49(5):965-974. doi:10.1249/MSS.0000000000001189

22. Labonté-LeMoyne É, Santhanam R, Léger P-M, Courtemanche F, Fredette M, Sénécal S. The delayed effect of treadmill desk usage on recall and attention. Computers in Human Behavior. 2015;46:1-5. doi:10.1016/j.chb.2014.12.054

23. Larson MJ, LeCheminant JD, Hill K, Carbine K, Masterson T, Christenson E. Cognitive and typing outcomes measured simultaneously with slow treadmill walking or sitting: implications for treadmill desks. PLoS One. 2015;10(4):e0121309. doi:10.1371/journal.pone.0121309

24. Creasy SA, Rogers RJ, Byard TD, Kowalsky RJ, Jakicic JM. Energy Expenditure During Acute Periods of Sitting, Standing, and Walking. J Phys Act Health. 2016;13(6):573-578. doi:10.1123/jpah.2015-0419

25. Burns J, Forde C, Dockrell S. Energy Expenditure of Standing Compared to Sitting While Conducting Office Tasks. Hum Factors. 2017;59(7):1078-1087. doi:10.1177/0018720817719167

26. Mansoubi M, Pearson N, Clemes SA, et al. Energy expenditure during common sitting and standing tasks: examining the 1.5 MET definition of sedentary behaviour. BMC Public Health. 2015;15:516. doi:10.1186/s12889015-1851-x

27. Saeidifard F, Medina-Inojosa JR, Supervia M, et al. Differences of energy expenditure while sitting versus standing: A systematic review and meta-analysis. Eur J Prev Cardiol. 2018;25(5):522-538. doi:10.1177/2047487317752186 